\title{
Review: reminiscence and life review are effective therapies for depression in the elderly
}

Bohlmeijer E, Smit F, Cuijpers P. Effects of reminiscence and life review on late-life depression: a meta-analysis. Int J Geriatr Psychiatry 2003;18:1088-94.

\section{What are the effects of reminiscence and life review in people with late life depression?}

\section{METHODS}

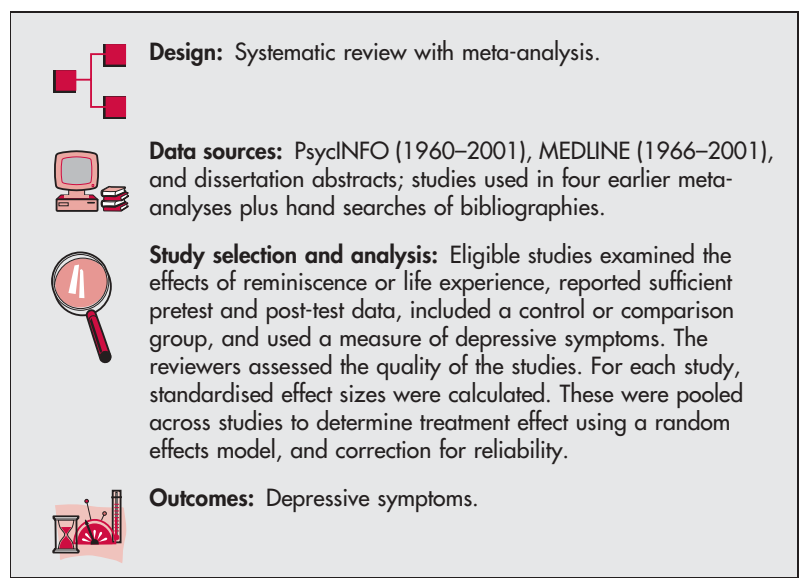

\section{MAIN RESULTS}

Twenty studies met inclusion criteria (15 RCTs, five comparative studies). Participants had severe depressive symptoms in five studies. In the other 15 studies, participants were not enrolled due to depression per se but had mild or moderate symptoms. Fifteen studies examined reminiscence and seven studies life review. Reminiscence and life review significantly improved depressive symptoms compared with control (large overall mean effect size $0.84, \mathrm{p}<0.001$, but significant heterogeneity was detected. The data were reanalysed omitting an outlier, resulting in a mean effect size of $0.67, \mathrm{p}<0.001)$. The effect size was larger in people with severe depression compared with mild or moderate depression $(\mathrm{d}=1.23 \mathrm{v}$ $\mathrm{d}=0.37$ ). There were no significant differences between reminiscence therapy and life review.

\section{CONCLUSIONS}

Reminiscence and life review were effective for elderly people, with similar effect sizes to well established drug and psychological treatments.

\section{NOTES}

Most included studies were small (range 21-201 participants); only $4 / 20$ studies were deemed to be high quality and dropout rates were high, four studies showing $>25 \%$. Also, there were insufficient data available to calculate long term effects.

For correspondence: Ernst Bohlmeiier, Department of Prevention Trimbos Institute, PO Box 725, 3500 AS Utrecht, the Netherlands; ebohlmeijer@ trimbos.nl

Sources of funding: none specified.
Commentary

$\mathrm{R}$ eminiscence therapy is, arguably, the only well developed intervention approach that can be said to be developmentally appropriate for older people. However, caution is needed in considering it as a unitary approach. Its diverse roots-ranging from psychodynamic therapy (the "life review") to oral history, for examplelead to many varieties of activity being subsumed under the popular umbrella of reminiscence. There is no agreed treatment protocol, and little agreement regarding its aims. It has been applied to a variety of populations, including people with dementia, where the body of evidence on its effectiveness is small. ${ }^{1}$ In relation to depression in older people, reminiscence outperformed other psychological therapies in a previous meta-analysis. ${ }^{2}$ The authors of the current review identify a number of relevant dimensions along which selected studies vary, including:

- modality (group $v$ individual)

- diagnosis (participants with a diagnosis of depression, or high scores on a self-report depression scale $v$ participants not selected on the basis of presence of depressive symptoms)

- type of reminiscence work (life review, involving a structured evaluative approach $v$ unstructured reminiscence)

- place of residence (community $v$ care home).

The review provides reassurance for the clinician in that effect sizes were higher for those with clinical levels of depression (perhaps because of floor effects on depression scales in non-clinical samples). Unfortunately the process by which reminiscence assists in liffing depressed mood remains unclear. Life review, with its emphasis on evaluation, resolution of past conflicts, and dealing with emotionally charged material, provides a framework for change, but did not perform significantly better than unstructured reminiscence. However, several of the "life review" studies adopted a group approach, whereas some would argue life review must occur individually. ${ }^{3} \mathrm{Knight}^{4}$ suggests that there is overlap between life review and classic approaches to grief work; it may be that with depression in later life often being concerned with loss, this provides a plausible therapeutic pathway. Knight also draws attention to possible negative outcomes, and future systematic reviews need to assess adverse effects as well as benefits.

Bob Woods, MA, MSc, CPsychol Dementia Services Development Centre, University of Wales Bangor, Bangor, Wales

1 Spector A, Orrell M, Davies S, et al. Reminiscence therapy for dementia: a review of the evidence of effectiveness (Cochrane review). In: The Cochrane Library, Issue 3. Oxford: Update Software, 1998.

2 Scogin F, McElreath L. Efficacy of psychosocial treatments for geriatric depression: a quantitative review. J Consult Clin Psychol 1994:62:69-74.

3 Haight BK, Dias JK. Examining key variables in selected reminiscing modalities. Int Psychogeriatr 1992;4(suppl 2):279-90.

4 Knight BG. Psychodynamic therapy with older adults: lessons from scientific gerontology. In: Woods RT, ed. Handbook of the clinical psychology of ageing. Chichester: Wiley, 1996:545-60. 